\title{
Comparison of the Antibacterial Efficiency and Effect on Root fracture Resistance and Bonding Strength Following Usage of Intracanal Disinfection Protocols (an In- vitro study)
}

\author{
Mohamed A. Sakr*, Ehab El- Sayed Hasanein**, Kareem \\ Moustafa El-batouty***, Waleed Mahmoud Eldars**** and \\ Shady Ali Hussein*****
}

\section{Abstract}

*Aim: to study the properties of 7 selected protocols for the disinfection of root canals in terms of their antibacterial effects against (Enterococcus faecalis), resistance of teeth used for fracture and the strength of bonding of obturation materials.

* Materials and methods : A total of 240 single rooted, single canaled teeth were selected for this study in accordance with pre-set conditions and were mechanically prepared and decoronated then randomly divided into three equal parts $(n=80)$ for each part of the research. The samples were randomly divided into 8 groups $(n=10)$ as following:Group 1 : Control group (No laser irradiation nor intracanal medication applied),Group 2 : Metapaste, Group 3 : Triple antibiotic paste (TAP), Group 4 : Ledermix, Group 5 : 940nm diode laser, Group 6 : 940nm laser irradiation followed by Metapaste application, Group 7 : Intracanal 940nm laser irradiation followed by (TAP) application, Group 8 : Intracanal 940nm laser irradiation followed by Ledermix application. In the first part of the study, samples were infected with E. Faecalis and incubated for 24 hours, then treated according to their group and incubated for one week after which quantification of the number of colonies after treatment was done. In the second part of the study, after the treatment of the samples, the root samples were then sectioned into cylindrical slices of $2 \mathrm{~mm}$ thickness to be tested for fracture resistance using universal testing machine. In the third part of the study,

* Asst. lecturer of endodontics, faculty of dentistry, Delta university for science and technology

** Professor of endodontics, faculty of dentistry, Ain shams university

*** Professor of endodontics, faculty of dentistry, Ain shams university

**** Assistant Professor of Microbiology and immunology, faculty of Medicine, Mansoura university

$* * * * *$ Lecturer of endodontics, faculty of dentistry, Ain shams university 
after the treatment of the samples, they were then obturated with gutta percha and $\mathrm{AH}$-plus sealer and after 2 days the root samples were sectioned into cylindrical slices thickness of $2 \mathrm{~mm}$ to perform Push-out test.All data were recorded, tabulated and statistically analyzed.

*Results: The results of the Antibacterial part of the study indicated that all the used disinfection protocols were capable of effectively disinfect the canals, Group 3 (TAP) showed the highest disinfection capability percentage of $\mathbf{( 9 6 . 4 \% )}$. The results of the Fracture resistance part of the study showed that all the used disinfection protocols were capable of effectively decrease the fracture resistance of the radicular dentin and a synergistic effect could be observed. The results of the Bond strength test, Group 2 (Metapaste) showed the highest posttreatment mean push-out strength values of (6.83 Mpa) compared to all treatment groups.

Conclusions: All used protocols were effective against the bacteria, TAP with 940nm diode laser was the most effective. All the protocols used negatively affected the resistance of the roots to fracture except laser radiation alone whose effect could be neglected. All the protocols used enhanced the bonding strength to obturation materials (gutta percha and resin-based $\mathrm{AH}$-plus sealer). The $940 \mathrm{~nm}$ Laser is a safer and promising option for intracanal disinfection that can be applied in conjunction with other intracanal disinfection means.

\section{Introduction}

The basic objective of root canal treatment (RCT) is the removal of the cause and treatment of signs and symptoms to avoid interappointment flare -ups and ensure successful root canal treatment especially in cases of necrotic pulp. E.faecalis is a common root canal pathogen in root canals with documented high resistance towards several antimicrobial agents.

AIM: The target of this study is to realize the antibacterial efficiency together with the effect on radicular dentin physical structure after intracanal procedures based on an antimicrobial strategy by usage of six different intracanal disinfection protocols.

\section{Materials and methods}

- This study was carried out in the Endodontics Department, Faculty of dentistry, Ain Shams University, Egypt. The study was approved by the Research ethics committee and planned to include three independent parts : 1) The Antibacterial Study, 2) Fracture resistance Study, and 3) Bond strength Study.

\section{${ }^{*}$ Collection, Selection and storage of teeth:}

- A total of 240 freshly extracted for orthodontic, periodontal or prosthetic reasons, single straight rooted, single canaled and sound maxillary and mandibular teeth were selected in refrence to specific inclusion and exclusion criteria:

1. Inclusion Criteria:_Single rooted and single canaled, Completely formed apices and Teeth without resorptive defects.

2. Exclusion criteria : Root caries, Heavily restored teeth, Previous root canal treatment. Cracked, fractured, resorped or severly curved roots and Narrow/calcified canals or internal resorption.

\section{* Mechanical preparation and disinfection of teeth: ${ }^{(1-7)}$}

- The selected teeth were decoronated at the level of Cemento enamel Junction Root specimens of standard length of $12 \mathrm{~mm} \pm 1 \mathrm{~mm}$ were obtained. The patency of the canals was then checked by ISO size $10 \mathrm{k}$-file, then the working length was determined by subtracting $1 \mathrm{~mm}$ to obtain the adjusted working length. The canals were then instrumented and enlarged to ISO size $25 \mathrm{k}$-file and Mechanical preparation was completed using Protaper rotary Files starting from Sx size and finishing by $\mathrm{F} 3$ size, with irrigation using $1 \mathrm{ml} 5.25 \%$ $\mathrm{NaOCl}$ alternative with $17 \%$ EDTA solution 
in between each file size and the other. Final preparation was done by Low speed post drill $(1.30 \mathrm{~mm})$ in diameter for the whole length of the roots creating canals of standardized volume and dimensions. ${ }^{(3-6)}$ and final irrigation of the canals was done using $5 \mathrm{ml}$ of $17 \%$ EDTA for 5 minutes, $5 \mathrm{ml}$ of $5.25 \% \mathrm{NaOCl}$ for 5 minutes respectively. This was followed by 30 min irrigation with distilled water to neutralize the effect of EDTA \& $\mathrm{NaOCl}$ solutions .Canals were then dried by ISO size 30 paper point and left to dry for 24 hours. The apical ends of root specimens were then sealed by temporary filling (Cavit,3M ,USA).All samples were the pouched in sterile sterilization pouches and autoclaved for 1 cycle of $121^{\circ} \mathrm{C}$ at $15 \mathrm{lbs}$. pressure for 15 minutes. The 240 prepared and disinfected samples were then equally and randomly divided onto the 3 parts ot the study $(n=80)$, each part was planned to be represented by 8 equal groups $(n=10)$ as following: Group 1 : Control group (No laser irradiation nor intracanal medication applied) ,Group 2 : Intracanal medication with Metapaste, Group 3 : Intracanal medication with Triple antibiotic paste (TAP), Group 4 : Intracanal medication with Ledermix ,Group 5 : Intracanal irradiation with $940 \mathrm{~nm}$ diode laser, Group 6 : Intracanal 940nm irradiation followed by Metapaste application, Group 7 : Intracanal 940nm irradiation followed by (TAP) application and Group 8 : Intracanal 940nm irradiation followed by Ledermix application.

\section{* Method of Laser irradition :}

- A diode laser device (Epic 940nm diode laser, Biolase) was used for this study ,being equipped with sterile endodontic flexible glass quartz fiber optic tips , $200 \mu \mathrm{m}$ in diameter and $14 \mathrm{~mm}$ in length, connected to a fiber optic cable. Their conical outline allowed radial emission of laser beam. ${ }^{(8-9)}$ In the device home screen, Endo mode was selected, and the laser setting used in the whole study was 1.0 Watts power, in the continuous mode. The maneuver of intracanal irradiation was a $5 \mathrm{sec}$ irradiation followed by a 20secinterval (to avoid temperature changes that could affect the physical properties of dentin), which constituted one lasing cycle. Four such cycles were performed for each tooth. With the root sample held by sterile tweezers , the tip was introduced $1 \mathrm{~mm}$ shorter than the apex, followed by activation during rotating the tip backward and forward apicocoronally and corono-apically at a speed of $2 \mathrm{~mm} / \mathrm{s}$ while touching the canal walls. This helicoidal (oscillatory) motion ensured that the whole canal walls were irradiated..$^{(3,5)}$ The application of the intracanal medications and laser irradiation was done in the same setting for all samples of all groups and with the same operator to avoid bias in results.

1. The Antibacterial Study.

a)Bacterial inoculum preparation (isolation and culturing):

- A pure standard Enterococcus Faecalis strain (ATCG 29212) was isolated and subcultured in blood agar plates and incubated at $37^{\circ} \mathrm{C}$ for 24 hours pure single E.faecalis colony was isolated from the cultured plates and gram stained to confirm the colony growth under microscope, then inoculated with nutrient broth and incubated again at $37^{\circ} \mathrm{C}$ for 24 hours in anaerobic conditions. The bacterial growth was checked by changes in the broth turbidity. A standardized suspension of turbidity adjusted spectrophotometrically to $0.5 \mathrm{Mc}$ - Farland units, equivalent to $1.5 \times 10^{8}$ colony forming units / milliliter (CFU/ml).

\section{b)Infection of dentin models:}

- Under aseptic conditions ,the lumen of the prepared roots were infected by the prepared bacterial suspension .Using a sterile micropipette with sterile tip, the lumen of the samples was injected with approximately 10 $\mu \mathrm{l}$ of the prepared bacterial suspension. ${ }^{(3-5)}$ The specimens were then incubated for 48 hours with nutrient broth at $37^{\circ} \mathrm{C}$, with daily replacement of the broth using a sterile micropipette with sterile tip to meet the needs of growing bacteria and avoid desiccation. ${ }^{(8)}$ For pre-treatment bacterial sampling, the root canals were filled with sterile saline as a transport fluid then, sterile ISO size 30 absorbent paper points absorbed the transport fluid for 60 seconds and transferred 
to a test tube containing $2 \mathrm{ml}$ of nutrient broth. All samples were vortexed for 30 seconds and 10-fold serial dilutions were prepared in saline. Aliquots of $0.1 \mathrm{ml}$ were spread plated onto blood agar plates, incubated at $37^{\circ} \mathrm{C}$ for 24 hours, and colony-forming units (CFU) per $1 \mathrm{ml}$ were counted by the same microbiologist and using the standard methods (magnifying lens under light microscope). ${ }^{(4,)}$ The Pre-treatment $\mathrm{CFU} / \mathrm{ml}$ values were recorded for each group specimens and tabulated.

c)Application of intracanal medications and laser irradiation of dentin models:

- The selected specimens were randomly divided onto 8 groups and intracanal medications and laser irradiation were applied into specimens for each treatment group as specified before. In groups (6-8), laser irradiation was done prior to application of the intracanal medications. After application of medications and/or laser irradiation, the samples of each group in addition to control group samples were sealed by temporary filling and placed in petri dishes with $2 \mathrm{ml}$ of nutrient broth, then the dishes were incubated for 1 week at $37^{\circ} \mathrm{C}$, with daily replacement of the broth using a sterile micropipette with sterile tip to meet the needs of growing bacteria and avoid desiccation. ${ }^{(5,6)}$ The 1 week incubation period was suggested to be sufficient to allow adequate bacterial growth and infection of dentinal tubules. ${ }^{(4,6)}$

d) Recovery and post- treatment sampling of bacteria from dentin models:

- Following the 1 week incubation period, intracanal medications were removed under complete aseptic conditions. The lumen of canals were irrigated with sterile saline and the medications were then removed by agitation with size $30 \mathrm{H}$-file. Post-treatment bacterial sampling was done as in pre-treatment sampling with the 10-fold serial dilution was maintained at $10^{3} \cdot{ }^{(4,8)}$ The Post-treatment $\mathrm{CFU} / \mathrm{ml}$ values were recorded for each group specimens and tabulated

2. The Fracture Resistance Study.

- A total of 80 randomly selected and mechanically prepared sterile root specimens for this part of the study were managed as following ${ }^{(1)}$ :

a) Preparation of root specimens:-The selected specimens were randomly divided onto 8 groups andintracanal medications and laser irradiation were applied into specimens for each treatment group as specified before. In groups (6-8), laser irradiation was done prior to application of the intracanal medications.After application of medications and/or laser irradiations, the samples of each group in addition to control group samples were sealed by temporary filling and stored physiological saline solution for 1 week at $37^{\circ} \mathrm{C}$,with replacement of the solution every 3 days to avoid dehydration. After the storage period, intracanal medications were removed. The lumen of canals were irrigated with sterile saline and the medications were then removed by agitation with size $30 \mathrm{H}$-file. The root specimens were then mounted in blocks of cold cured acrylic resin. The blocks were marked with an indelible marker. Using the diamond disk with low speed and water coolant, root specimens with in the acrylic blocks were sectioned horizontally, in coronoapical direction and perpendicular on the root canal, creating dentin discs of $2 \mathrm{~mm}$ thickness confirmed by a ruler. The cervical $2 \mathrm{~mm}$ thick dentin discs were selected for fracture resistance test using the universal testing machine, irrigated with saline to remove any debris and stored in physiological saline till time of testing.

\section{b) Fracture resistance testing: (Fig. 1)}

- The root canal access at the coronal side of the prepared cervical dentin discs was shaped using water cooled low speed carbide bur creating a coronal root seat to receive the spherical tip of the loading punch of the universal testing machine. Vertical loading force was applied at a cross head speed of $1 \mathrm{~mm} / \mathrm{min}$ until the root cylinder was fractured.The peak load at fracture was automatically recorded by the machine software and expressed in Newtons (N). Peak load values for each group specimens were tabulated. 


\section{The Bond strength Study.}

- A total of 80 randomly selected and mechanically prepared sterile root specimens for this part of the study were managed as following:

\section{a) Preparation of root specimens:}

- The selected specimens were randomly divided onto 8 groups and intracanal medications and laser irradiation were applied into specimens for each treatment group as specified before.In groups (6-8), laser irradiation was done prior to application of the intracanal medications. After application of medications and/or laser irradiations, the samples of each group in addition to control group samples were sealed by temporary filling and stored physiological saline solution for 1 week at $37^{\circ} \mathrm{C}$, with replacement of the solution every 3 days to avoid dehydration. After the storage period, intracanal medications were removed. The lumen of canals were irrigated with sterile saline and the medications were then removed by agitation with size $30 \mathrm{H}$-file.

b)Obturation and sectioning of the root specimens:

- For obturation of the specimens, the master F3 protaper gutta percha cone after being checked for fitness in the prepared canals. Samples were then stored for 48 hours in an incubator at $37^{\circ} \mathrm{C}$ in $100 \%$ humidity for complete setting of the sealer.The root specimens were then mounted in blocks of cold cured acrylic resin. The blocks were marked with an indelible marker .Using the diamond disk with low speed and water coolant, root specimens with in the acrylic blocks were sectioned horizontally, in coronoapical direction and perpendicular on the root canal, creating dentin discs of $2 \mathrm{~mm}$ thickness confirmed by a ruler. The cervical $2 \mathrm{~mm}$ thick dentin discs were selected for fracture resistance test using the universal testing machine Both apical and coronal aspects were photographed before the test to confirm circular canal space and that the sealer filled the entire canal space together with the gutta percha with absence of voids. If otherwise, the defective samples, were excluded and replacement teeth sections were prepared in the same manner $(3 \pm 4$ samples excluded from each group). The obtained discs were ground on both sides with the diamond disc to make the restoration flushing with the dentin surface.

\section{D) Bond strength (Push- out) testing: ${ }^{(9)}$}

- The $1.20 \mathrm{~mm}$ diameter metallic punch was used in the Push-out test, to provide almost complete coverage over the gutta percha mass $(1.30 \mathrm{~mm}$ in diameter) without touching the canal walls as the plunger was positioned with a clearance of approximately $0.1 \mathrm{~mm}$ from the margins of the dentinal wall of the canal. This was done to ensure contact with only the obturation material. Vertical loading force was applied at a cross head speed of $1 \mathrm{~mm} / \mathrm{min}$ until the Bond failure occurred. Failure manifested by the extrusion of the obturation material and confirmed by sudden drop along load-strain curve recorded by computer software .The peak load at bond failure was automatically recorded by the machine software and expressed in Newtons (N). Push Out strength was calculated in Megapascals(Mpa) and results for each group were tabulated using the formula: Push- out strength (Mpa) = $F / A$, Where, $F=$ peak load at bond failure in Newtons,$A=$ Curved surface area of the testing specimen(cylindrical disc) in $\mathbf{m m}^{2}=$ $(2 \pi r h)$, With,$\pi=$ constant $=3.14, r$ - radius of the central hole, $h$ - thickness of specimen

\section{$\underline{\text { Results }}$}

* For all groups in the three parts of the study, the tabulated data were presented as means, standard deviation (SD), standard error (SE), minimum values and maximum values. All of the quantitative data were analyzed statistically using IBM SPSS Statistics for Windows, version 20.0 (IBM Corp., Armonk, NY, USA). One way analysis of variance (ANOVA) was used for data analysis, whenever a statistical significant difference was recorded, pairwise comparisons between tested groups were performed using Tukey's post-hoc test. For all tests, the P-value of 0.05 or less was considered for statistical significance. 
Part (a): Antibacterial effect against E. feacalis.

- The antibacterial effect was represented as the mean bacterial count (CFU/ml) for each group.Pre- treatment sampling and statistical analysis was done to ensure non- significant difference between groups regarding the initial bacterial count before application of intracanal disinfection protocols to avoid bias. The post-treatment sampling statistical analysis expressed the effect of each disinfection protocol in addition to the effectiveness of disinfection ability compared to the posttreatment control group. The percentage of bacterial load reduction (disinfection capability percentage) following the use of the treatment was calculated in reference to the post treatment bacterial count in control group. (Fig.2).

- The specimens of the positive control group (Group1) showed higher statistically significant post-treatment mean $\mathrm{CFU} / \mathrm{ml}$ values compared to post treatment CFU/ $\mathrm{ml}$ values for all the treatment groups. Comparing the treatment groups in which intracanal medications were used without laser,(Groups 2,3,4) ; Group 2 (Metapaste) showed the highest mean $\mathbf{C F U} / \mathbf{m l}$ value and least disinfection capability percentage of (76.03\%) followed by Group 4 (Ledermix) with disinfection capability percentage of (93.7\%) and then Group 3 (TAP) that showed the least mean $\mathrm{CFU} / \mathrm{ml}$ value and highest disinfection capability percentage of $(96.4 \%)$. The difference was statistically significant in Group 2 (Metapaste) if compared to Group 3 (TAP), Group 4 (Ledermix) but, was not statistically significant between Group 3 (TAP) and Group 4 (Ledermix). The Group 5 (Laser irradiation only) specimens showed the highest statistically significant mean CFU/ $\mathbf{m l}$ values among all treatment groups, with the least disinfection capability percentage of $(\mathbf{3 6 \%})$.Considering the treatment groups in which intracanal medications were used with laser irradiation, Groups 6,7,8 ; Group 7 (Laser/ TAP) showed the lowest mean CFU/ $\mathbf{m l}$ value among all the treatment groups and highest disinfection capability percentage of
(98\%), followed by Group 8 (Laser/Ledermix) with disinfection capability percentage of (96.7\%) and then Group 6 (Laser/Metapaste) that showed the highest mean CFU/ml value and lowest disinfection capability percentage of $(\mathbf{9 5 . 7 \% )}$. The difference was statistically significant in Group 6 (Laser/ Metapaste) if compared to Group 7 (Laser/ TAP) , Group 8 (Laser/Ledermix) but ,was not statistically significant between Group 7 (Laser/ TAP) and Group 8 (Laser/ Ledermix).Comparing the treatment groups in which intracanal medications were used without laser irradiation , Groups 2,3,4; with treatment groups in which the same intracanal medications were used with laser irradiation, Groups 6,7,8; there were differences in the mean $\mathrm{CFU} / \mathrm{ml}$ values and disinfection capability percentages, as Laser / intracanal medicated Groups 6,7,8 showed generally lower mean $\mathrm{CFU} / \mathrm{ml}$ values and higher disinfection capability percentages than the corresponding groups treated with the intracanal medication alone. The difference was statistically significant only in between Group 2 (Metapaste) if compared to corresponding Group 6 (Laser/ Metapaste).

\section{Part (b): Radicular dentin fracture} resistance test results.

- The Radicular dentin fracture resistance were represented as the mean peak load values in Newtons $(\mathbf{N})$ at fracture that was automatically recorded by the machine software during testing the specimens of each group using the Universal testing machine. The highest mean peak load value was for the specimens of the positive control group $($ Group 1$)=(235.52 \mathrm{~N})$, and the lowest mean peak load value was for the specimens of $940 \mathbf{~ n m ~ d i o d e ~ l a s e r / ~ T A P ~ g r o u p ~ ( G r o u p ~}$ $\mathbf{7})=(\mathbf{8 7 . 3 6} \mathbf{N})$. The specimens of the positive control group (Group1) showed higher significant post-treatment mean peak load values at fractures in Newtons $(\mathbf{N})$ compared to all the treatment groups. The difference was statistically significant for all groups except for Group 5 (Laser irradiation only). The Group 5 (Laser irradiation only) specimens showed 
the highest statistically significant posttreatment mean peak load value at fracture among all treatment groups of $(\mathbf{2 2 7 . 1 1} \mathbf{N})$. Considering the treatment groups in which intracanal medications were used without laser, (Groups 2,3,4) showed differences in the post-treatment mean peak load values, as, Group 2 (Metapaste) showed the highest post-treatment mean peak load value of $(130.89 \mathrm{~N})$ followed by Group 3 (TAP) with mean peak load value of (118.01N) and then Group 4 (Ledermix) that showed the lowest mean peak load value of (117.1N). Although this difference in values, they were statistically non-significant.Comparing the treatment groups in which intracanal medications were used with laser irradiation, Groups 6,7,8; specimens showed different post-treatment mean peak load values but this difference was not statistically significant. Group 7 (Laser/ TAP) showed the lowest post-treatment mean peak load value of (87.36N) among all the treatment groups, followed by Group 8 (Laser/ Ledermix) of (95.02N) and finally Group 6 (Laser/ Metapaste) that showed the highest post-treatment mean peak load value of $(\mathbf{9 5 . 1 1} \mathbf{N})$.Comparing the treatment groups in which intracanal medications were used without laser irradiation , Groups 2,3,4; with treatment groups in which the same intracanal medications were used but with laser irradiation, Groups 6,7,8; there were differences post-treatment mean peak load values which were lower for the groups with combined laser and intracanal medication treatment than the corresponding groups treated with the intracanal medication alone. These differences were statistically significant except in between Group 3 (TAP) , Group 7 (Laser/TAP), where the difference was not statistically significant

\section{Part (c): Push-out test results.}

- The push-out Strength (bond strength) values were represented as the mean peak load values in Megapascals (Mpa) at bond failure (dislodgment of obturation material) that was automatically recorded by the machine software during testing the specimens of each group using the Universal testing machine. The lowest push-out strength value was for the specimens of the positive control group $($ Group 1$)=(2.82 \mathrm{Mpa})$, and the highest push-out strength value was for the specimens of Metapaste (Group 2)= $(6.83 \mathrm{Mpa})$.Considering the treatment groups in which intracanal medications were used without laser, (Groups 2,3,4); they showed statistically significant differences in the posttreatment mean push-out strength value, as, Group 2 (Metapaste) showed the highest post-treatment mean push-out strength value of (6.83 Mpa) compared to all treatment groups. Group 3 (TAP) showed slightly statistically significant higher mean push-out strength value of (4.98 Mpa) than Group 4 (Ledermix) that showed the mean push-out strength value of (4.23 Mpa). Group 5 (Laser irradiation only) showed the least post-treatment mean push-out strength value of (3.47 Mpa) among all treatment groups.Comparing the treatment groups in which intracanal medications were used with laser irradiation, Groups 6,7,8; specimens showed statistically significant difference in mean push-out strength values, where Groups 6 (Laser/Metapaste) showed the highest mean push-out strength value of (6.81 Mpa) followed by Group 7 (Laser/TAP) with mean push-out strength value of (5.19 Mpa), and then Group 8 (Laser/Ledermix) with mean push-out strength value of $\mathbf{( 4 . 3 2}$ Mpa). Comparing the treatment groups in which intracanal medications were used without laser irradiation , Groups 2,3,4 ; with treatment groups in which the same intracanal medications were used but with laser irradiation, Groups $\mathbf{6 , 7 , 8}$; there were differences in mean push-out strength values which were slightly higher for the groups with combined laser and intracanal medication treatment than the corresponding groups treated with the intracanal medication alone. These differences were statistically significant except in between Group 2 (Metapaste) , Group 6 (Laser/Metapaste) , where the difference was not statistically significant. 


\section{Discussion:}

The protocol for teeth selection, storage and preparation was adapted from previous similar studies and standardized for all the current study parts to avoid bias in results. The idea for usage of a peso reamer for final shaping of the canals was to create dimensionally standardized canal space for identical canals volume that ensured standardized bacterial suspension content and standardized dimensions of obturation mass within the canals during the antibacterial and Push-out tests respectively. ${ }^{(1-6)}$ The application of Intracanal medications in all groups of the 3 parts of the study followed also a standardized protocol, and according to the followed steps discussed in previous literatures. ${ }^{(1,4,7)}$

Regarding the antibacterial part of this study, the choice of $\mathrm{E}$. faecalis was related to the reported resistance of these bacteria to most of the intracanal irrigation materials and medications. Many antimicrobial studies evaluated the antibacterial effect of various materials used in endodontic treatment procedures including intracanal medications and laser irradiations with variable parameters against E. faecalis. The results of the Antibacterial part of the study indicated that all the used disinfection protocols were capable of effectively disinfect the canals and that in absence of intracanal medications, extensive growth of residual bacteria within canals can occur as evident by higher post-treatment mean CFU $/ \mathrm{ml}$ values compared to post treatment values for all the treatment groups .It this study, comparing the treatment groups in which intracanal medications were used without laser , (Groups 2,3,4) , Group 3 (TAP) showed the highest disinfection capability percentage of $\mathbf{( 9 6 . 4 \% )}$ followed by Group 4 (Ledermix) with disinfection capability percentage of $\mathbf{( 9 3 . 7 \% )}$ proved to be more effective than Group 2 (Metapaste) with least disinfection capability percentage of $(\mathbf{7 6 . 0 3} \%)$. The results also showed that the $940 \mathrm{~nm}$ laser irradiation alone (Group 5) was capable of disinfection of the canals even if with with the least disinfection capability percentage of $\mathbf{( 3 9 \% )}$ amoung all treatment groups. This agreed with a previous study reported by Ruth et al.(2017) $)^{(10)}$, which concluded that the usage $940 \mathrm{~nm}$ diode laser in conjugation with Radial firing tips showed a satisfactory bactericidal effect without any thermal side effect to the tooth-supporting tissues.

The results demonstrated that upon comparing the treatment groups in which intracanal medications were used with laser irradiation, (Groups 6,7,8) with treatment groups in which intracanal medications were used without laser (Groups 2,3,4) ,or laser irradiation alone (Group 5), a synergistic effect could be observed, between the antibacterial effect of laser and intracanal medications that surpass the effect of either of them if used alone.The nature of this synergism may be due to additive bactericidal effect of laser irradiation to the effect of the intracanal medication or may be attributed to some sort of physical or chemical interaction. Due to limited similar studies about this issue, further studies should be conducted to evaluate the nature of this synergism.

The results of the Fracture resistance part of the study showed that all the used disinfection protocols were capable of effectively decrease the fracture resistance of the radicular dentin . The difference was statistically significant for all groups except for Group 5 (Laser irradiation only). This indicated that all used intracanal disinfection protocols adversely affected the radicular dentin fracture resistance except for intracanal laser irradiation, whose effect can be neglected if compared to other treatment groups. Yassen et al.(2013) $)^{(1)}$ investigated the effect of intracanal medications on root fracture resistance and concluded that The application of the used medications decreased the fracture resistance at all time points .In this study, comparing the treatment groups in which intracanal medications were used without laser , (Groups 2,3,4) , all the used medications adversely affected the radicular dentin fracture resistance without significant difference after 1 week application. The results showed that although the $940 \mathrm{~nm}$ laser irradiation alone (Group 5) slightly decreased the fracture resistance value relative to positive control group, but this difference was insignificant 
. Comparing the treatment groups in which intracanal medications were used with laser irradiation, (Groups $\mathbf{6 , 7 , 8}$ ) with treatment groups in which intracanal medications were used without laser (Groups 2,3,4), or laser irradiation alone (Group 5), a synergistic effect could be observed, especially in Groups 6 (Laser/Metapaste) , 8 (Laser/Ledermix) - Due to limited similar studies about this issue, further studies should be conducted to evaluate the nature of this synergism.

The effect of different intracanal medications on the intracanal bond strength to different obturation material was evaluated in many previous studies In the present study, all used intracanal disinfection protocols enhanced the radicular dentin bond strength to used obturation materials but with variable degrees. Considering the treatment groups in which intracanal medications were used without laser, Group 2 (Metapaste) showed the highest post-treatment mean push-out strength values of $(\mathbf{6 . 8 3} \mathbf{M p a})$ compared to all treatment groups. Group 3 (TAP) showed slightly statistically significant higher mean push-out strength values of (4.98 Mpa) than Group 4 (Ledermix) that showed the mean push-out strength values of (4.23 Mpa).

In this study, although different wavelength and power settings (940nm,1 Watts) was used for diode laser irradiation compared to previous studies, Group 5 (Laser irradiation only) results indicated that laser irradiation has a positive effect on the bond strength even if it had showed least post-treatment mean pushout strength values of among all treatment groups. The results of the bond strength showed also existing synergism between the effect of laser and both TAP and Ledermix intracanal medications that surpass the effect of either of them if used alone.Due to limited literature resources about the effect of 940nm on either bond strength or fracture resistance of radicular dentin, further studies are recommended to evaluate its effect with different parameters. Also, the nature of the existed synergism between the effect of laser and intracanal medications observed in Groups 7(Laser/TAP) \& 8 (Laser/ledermix) should be evaluated.
Correlating the results of the three parts of the study, it can be stated that no disinfection protocol is ideal, as for example TAP paste either alone or combined with laser, presented the highest disinfection ability ,but in the same time significantly affected the radicular dentin resistance to fracture although increasing the bond strength to radicular dentin.

Conclusion : Within the confines and limitations of this study, the proposed null hypothesis could be rejected, as the used disinfection protocols had variable effects on E.faecalis and biomechanical properties of dentin. All used protocols were effective against the bacteria, TAP with $940 \mathrm{~nm}$ diode laser was the most effective.All the protocols used negatively affected the resistance of the roots to fracture except laser radiation alone whose effect could be neglected.All the protocols used enhanced the bonding strength to obturation materials (gutta percha and resin-based (AH-plus sealer) . The $940 \mathrm{~nm}$ diode Laser is a safer and promising option for intracanal disinfection that can be applied in conjunction with other intracanal disinfection means.

Recommendations:1) Other laboratory studies to assess the effect of the same protocols used with other strains of bacteria ,2) Other laboratory studies to evaluate the antibacterial effect and effect on physical and mechanical properties of root tissue of laser radiation $(940 \mathrm{~nm})$ with different parameters either alone or in addition to other protocols ,3) Clinical studies to assess the effect of the same protocols. 


\section{Refrences:}

1)Yassen H., Vail M., Chu P., latt A. The effect of medicaments used in endodontic regeneration on root fracture and micro hardness of radicular dentine. International Endodontic Journal. 2013;46:688-95.

2)Nagas E., Cehreli C., Uyanik O., Vallittu P., Lassila J. Effect of several intracanal medicaments on the push-out bond strength of ProRoot MTA and Biodentine. International Endodontic Journal.2015;48:1-7.

3) Kaiwar A., Usha H., Meena N. ,Ashwini P., Murthy S. The efficiency of root canal disinfection using a diode laser: In vitro study. Indian J Dent Res.2013;24:14-8.

4) Shokraneh A., Farhad A., Saatchi M., Mohsen S . Antibacterial effect of triantibiotic mixture versus calcium hydroxide in combination with active agents against Enterococcus faecalis bioflm. Dent Mater J 2014; 33:733-38.

5) Naghavi N., Rouhani A., Irani S., Banihashemi E. Diode Laser and Calcium Hydroxide for Elimination of Enterococcus Faecalis in Root Canal. JDMT.2014;3:55-60.

6)Gordon W., Atabakhsh V., Meza F., Doms A., Nissan R., et al. The antimicrobial efficacy of the erbium, chromium:yttrium-scandiumgallium garnet laser with radial emitting tips on root canal dentin walls infected with Enterococcus faecalis. JADA. 2007;138:9921002.

7)Shakouie S., Shahi S., Samiei M., Milani A., Reyhani M., et al. Effects of different intra canal medicaments on the push out bond strength of endodontic sealers. J Clin Exp Dent.2017;9:443-7.

8) Kanumuru N., Subbaiah R. Bacterial Effcacy of $\mathrm{Ca}(\mathrm{oH}) 2$ Against E.faecalisCompared with three Dental Lasers on Root Canal Dentin- An In vitro Study.Journal of Clinical and Diagnostic Research.2014;8:135-37.
9)Akman M., Akbulut M, Guneser B., AycE E. Effect of intracanal medicaments on the push-out bond strength of Biodentine in comparison with Bioaggregate apical plugs, Journal of Adhesion Science and Technology.2015;2:12-9.

10) Ruth S., Norbert G., Georg C., Rene F. The Impact of a $940 \mathrm{~nm}$ Diode Laser with Radial Firing Tip and Bare End Fiber Tip on Enterococcus faecalis in the Root Canal Wall Dentin of Bovine Teeth: An In Vitro Study. Photomedicine and Laser Surgery.2017,35: 357-63.
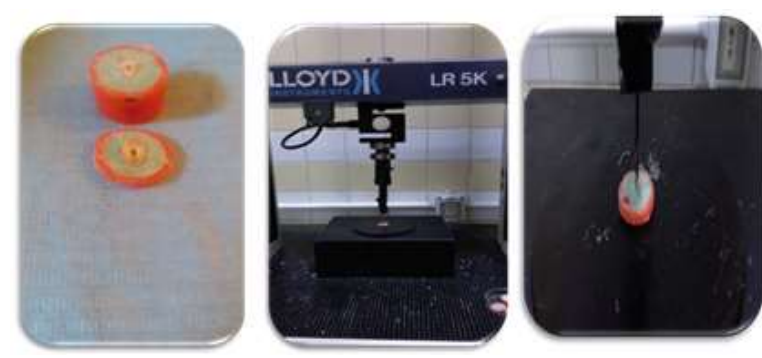

Figure (1) : $2 \mathrm{~mm}$ thick cervical section ready for fracture resistance test with the universal testing machiene

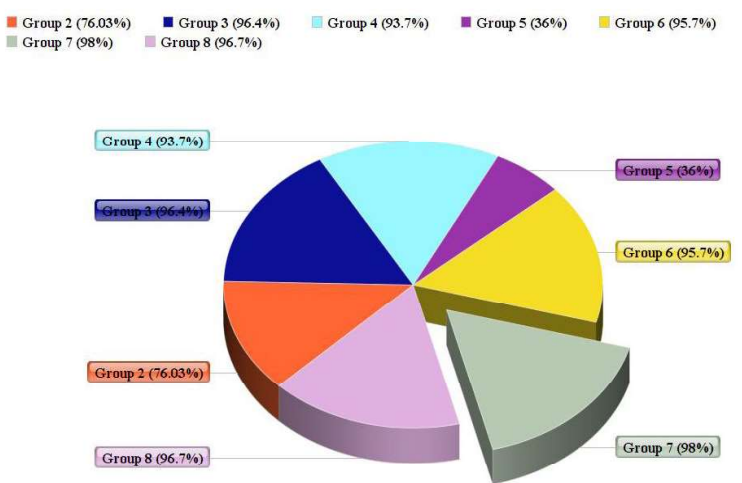

(Figure.2) : A pie chart comparing the disinfection capability for all groups. 